\title{
Hermaphroditism: an obsolete diagnosis?
}

\author{
Anastasios Tranoulis, Lina Michala \\ 1st Department of Obstetrics and Gynaecology, University of Athens, Medical School, General Hospital "Alexandra", \\ Athens, Greece
}

Dear Sir,

Our aim was to assess to what extent the traditional term 'hermaphrodite' is still in use to describe 'disorders of sex development' (DSD) as per the terminology recommended by the 2006 Chicago Consensus Statement. For this purpose we systematically searched Pubmed and Scopus using the keywords hermaphroditism, hermaphrodite, pseudohermaphrodite, pseudohermaphroditism and intersex in the title and abstract. All prospective and retrospective observational cohort studies and case reports published between 2007-2015 using the old terminology were included. A total of 161 articles meeting the inclusion criteria were identified. The vast majority of the articles were case reports published during the period 2007-2009. The first author was frequently affiliated to a genetic or an internal medicine department and $55 \%$ of the articles were published by university or tertiary centres. We thus conclude that the use of the old nomenclature is significantly diminishing

Key words: DSD, Disorder of Sex Development, Hermaphrodite, Intersex, Pseudohermaphrodite

Address for correspondence:

Lina Michala MRCOG, PhD, 1st Department of Obstetrics and Gynaecology, University of Athens, Medical School, "Alexandra", 80 V. Sofias Avenue, P.C. 11528 Athens, Greece; Tel.: +30 21077785535 , Fax: +30 2132162898,

E-mail: linamichalas@hotmail.com

Received: 20-02-2017, Accepted: 06-03-2017 especially from 2010 on. This depicts a progressive but not complete implementation of the revised terminology in accordance with the recommendations of the Chicago Consensus Statement.

\section{INTRODUCTION}

In 2006, a group of specialists in the management of disordered sexual development published a consensus statement which included the introduction of a new terminology and categorisation of such disorders. ${ }^{1}$ It left behind previous terms such as hermaphroditism, pseudohermaphroditism and intersex, which were deemed by the authors as anachronistic, derogatory and stigmatising to the patients and usually confusing for the doctors, and adopted the term Disorder of Sex Development, which was further categorised according to the chromosomal sex of the patient. ${ }^{1}$

Although initial reports suggested an acceptance of the new terminology, the impression gained from several publications is that the previous terms continue to be randomly used among the scientific community. ${ }^{2}$ The aim of this study was to objectively assess how often this terminology is used in peer reviewed publications and to assess its provenance and the trend in its usage over time.

\section{METHODOLOGY AND RESULTS}

We systematically performed a Pubmed and Scopus search using the keywords hermaphroditism, hermaphrodite, pseudohermaphrodite, pseudohermaphroditism and intersex in the title and abstract. 
The search was limited to articles after 2007, one year following the publication of the consensus statement, and only English language articles were included. All the prospective and retrospective observational cohort studies as well as case reports meeting the abovementioned criteria were included in the study.

We counted the number of articles published per year since 2007 and identified the type of article (case report, case series, review article or intervention study). We also checked the provenance of the articles to categorise them per specialty of leading author and country of origin. For the time period between 2007-2008 we further assessed what proportion of articles were using both terms in the abstract with a view to evaluating whether an attempt was being made to transition from the old to the new nomenclature. We identified 161 articles. Ninety-three (57.8\%) were case reports, 35 case series $(21.7 \%)$ and 33 review articles $(20.5 \%)$. Figure 1 shows the number and type of articles published per year. Eighty-eight (55\%) articles were published by university hospitals. The first author was affiliated to a genetic department in 20/161 cases, to an internal medicine department in 20/161 cases, while 19/161 were from a urology department, 17/161 were in a gynaecological and 19/161 were in a paediatric department.

As shown in Table 1, the majority of articles originated from North America (28/161), of which 25 were from the USA and three from Canada. A significant proportion originated from India (27/161) and China
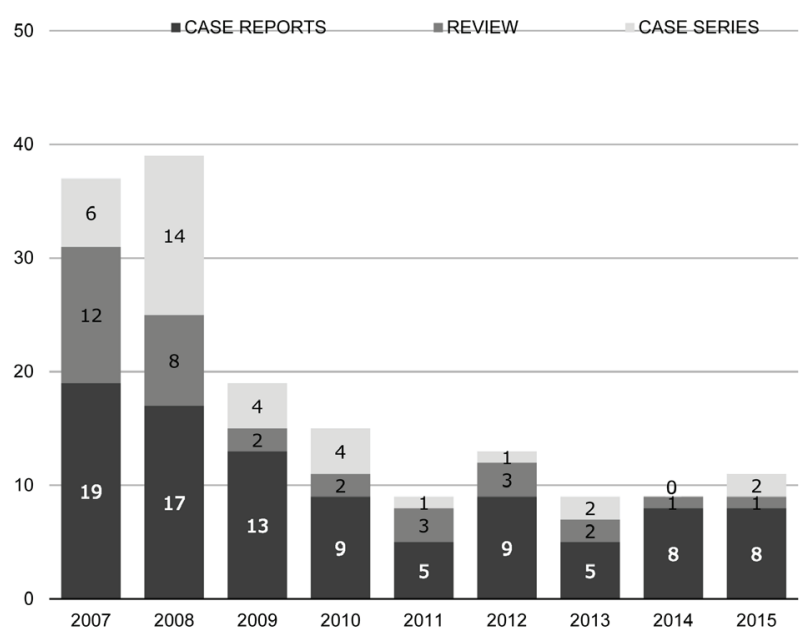

Figure 1. Number and type of articles per annum.
Table 1. Country of article' origin.

\begin{tabular}{lc}
\hline Country of origin & Number of articles \\
\hline India & 26 \\
USA & 25 \\
China & 18 \\
Turkey & 10 \\
Iran & 6 \\
Italy & 6 \\
Australia & 5 \\
Japan & 5 \\
Spain & 5 \\
Germany & 4 \\
Others & 51 \\
\hline
\end{tabular}

(18/161). Between 2007 and 2008, 13 of the articles found in our search were also identified in a search using the keyword DSD. Six of the articles (of which two were case reports and four reviews of the literature) interchanged the two terms, four articles mentioned in passing the old terminology and three articles were written specifically to present and explain the change in nomenclature.

\section{DISCUSSION}

The aim of this study was to evaluate the implementation of the proposed revised nomenclature and to assess the tendency to continue the use of the traditional terms. We have found that until recently, a significant proportion of authors have not been using the revised nomenclature for DSD in their publications. Most articles published soon after the publication of the consensus statement showed a predictable delay in the dissemination of the revised terminology. On the other hand, one year after the consensus statement, some of the articles were indeed using both terms interchanging them in the text, while others comprised reviews or articles specifically written with a view to enhancing implementation of the new terminology by clearly stating that the preferred terminology is henceforth DSD.

Our findings are in contrast to those of Pasterski 
et al, ${ }^{2}$ who in 2009 examined clinical management of DSD through a questionnaire distributed to tertiary paediatric centres across Europe and found that all centres were adhering to the new nomenclature. The same authors, however, also scrutinized the literature, sampling papers two years before and two years after the publication of the consensus statement. Although they identified a sharp drop in the use of the word intersex as a diagnostic descriptor, the terms hermaphroditism and pseudohermaphroditism were only slightly decreased, from 34 to $26 \%$ and from 35 to $19 \%$, respectively. ${ }^{2}$

The vast majority of patients with DSD are dealt with by paediatric endocrinologists and paediatric urologists who as a group have been widely informed about the guidance of the consensus statement through their professional bodies, seminars and conferences. This was reflected in our study, where the largest proportion of papers using the misnomers were written by internists and geneticists, specialists less likely to be involved clinically in the care of persons with DSD. Most non-specialists will only come across a limited number, if any, of DSD cases in their career and would be keener to publish a case report of the patient in their care to present it as a rarity. This would also explain the fact that more than half of the papers identified in our study were case reports.

Furthermore, there was an uneven geographic distribution of publications. The majority of articles using the misnomers were from North America (USA and Canada), probably indicating fewer referrals to tertiary centres of excellence in these countries. This may be due to a lack of centralised care in DSD, which in turn would not allow for awareness to aggregate within certain institutions. The second largest group of articles was from countries in Asia, most likely due to the fact that scientists from these countries were not represented at the Chicago consensus. Articles from Europe were less likely to use inadequate terminology, indicating embracement of the recommendations more widely and sooner.

We fear that lack of knowledge of the new nomenclature well after the years of the consensus publication may, moreover, reflect ignorance of the remaining recommendations of the statement. The thought that some DSD patients may be cared for by clinicians with anachronistic knowledge and suboptimal training is unsettling, especially when life-changing decisions about sex assignment, genital operations and diagnosis disclosure need to be made. DSD management through a multidisciplinary team, including a paediatric endocrinologist, a paediatric surgeon/ urologist, plastic surgeons, paediatric psychiatrists/ psychologists, gynaecologists, clinical geneticists, histopathologists and neonatologists, was one of the major suggestions of the Consensus statement. Meetings involving all related specialists within an institution could facilitate dissemination of information among other groups of specialists who may only be indirectly involved in DSD management. Discussions in such a setting would improve the awareness of all those involved in the care of DSD patients. Ideally, all teaching hospitals dealing with DSD should have in place a multidisciplinary team that would meet at regular intervals. Nevertheless, about half of the papers located in our study were from university hospitals, implying that there is a deviation from optimal management in many settings and that misinformation is still being passed on to a future generation(s) of physicians.

DSD are one of a small number of medical entities whose descriptive terminology has changed often and drastically over the past fifty years. Given the rarity of the conditions, a time lag would be expected before terms such as hermaphroditism, pseudohermaphroditism and intersex would disappear from the literature. However, to speed up the transition, specialists in the field of DSD should filter the terminology used in newer editions of medical textbooks and educational material in order to eliminate inadequate terms and educate the new generation of doctors appropriately.

\section{FUNDING}

No conflict of interest or financial support declared.

\section{REFERENCES}

1. Hughes IA, Houk C, Ahmed SF, et al, 2006 Consensus statement on management of intersex disorders. Arch Dis Child 91: 554-563.

2. Pasterski V, Prentice P, Hughes IA, 2010 Impact of the consensus statement and the new DSD classification system. Best Pract Res Clin Endocrinol Metab 24: 187-195. 